\title{
APPliCation OF BoX-JenKins Methodology in ELECTRIC CONSUMPTION FORECASTING of A Metallurgical EnTERPRise
}

\author{
Gulnara Ibrayeva, Iosif Breido \& Juliya Bulatbayeva
}
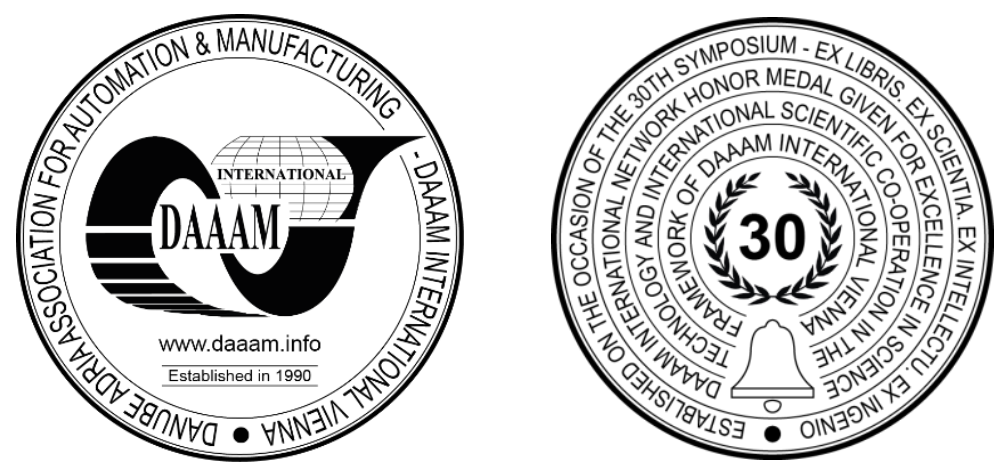

This Publication has to be referred as: Ibrayeva, G[ulnara]; Breido, I[osif] \& Bulatbayeva, J[uliya] (2021). Application of Box-Jenkins Methodology in Electric Consumption Forecasting of a Metallurgical Enterprise, Proceedings of the 32nd DAAAM International Symposium, pp.0493-0499, B. Katalinic (Ed.), Published by DAAAM International, ISBN 978-3-902734-33-4, ISSN 1726-9679, Vienna, Austria

DOI: $10.2507 / 32$ nd.daaam.proceedings.071

\begin{abstract}
The article discusses the construction of a predictive model of power consumption of a large industrial facility based on the Box-Jenkins methodology. This method belongs to the tools for statistical data processing. Retrospective data on power consumption of the metallurgical enterprise JSC "ArcelorMittal Temirtau" were taken as the initial data for forecasting. The graphs of the initial time series, autocorrelation function and private autocorrelation function are presented. Based on the analysis of auxiliary graphs, alternative parameters of the predictive model are selected. Among the results of the experiment, the best parameters of the model were selected based on the assessment of adequacy and accuracy.
\end{abstract}

Keywords: short-term forecasting; time series analysis; autocorrelation function; ARIMA model; power consumption of the metallurgical plant; statistical analysis.

\section{Introduction}

The laws of modern wholesale electricity markets in post-Soviet countries are undergoing constant changes, which puts new demands on its participants. Such transformations include the introduction of a balancing electricity market, the integration of which is expected in 2022 in Kazakhstan. The balancing market principle can be described as follows. To connect them to the national electric grid, consumers submit hourly applications for the planned consumption of electric energy every day in advance. The claims represent a daily electrical load profile. According to the Law on the Electricity Industry of the Republic of Kazakhstan, large consumers with a load capacity of more than 1 MW are considered to be participants in the wholesale electricity market. After the end of each operational day, the System Operator of the electrical network monitors the planned and actual consumption. For each hour when the consumer has exceeded the declared capacity, it is necessary to pay the difference at the inflated tariff. Thus, in order to incur lower financial losses, the consumer must minimize the difference between the declared and actual consumption. 
This can be achieved by managing the electricity consumption schedule or more accurately forecasting the declared consumption. However, managing the schedule of electricity consumption in large enterprises, especially with different types of production, is a difficult task. Therefore, consumers need to analyse their own power consumption and create working forecasting models.

There is no unambiguously suitable model among the methods for forecasting electricity consumption. For each individual enterprise, an analysis should be carried out, alternative models should be built and the optimal model should be chosen. Hourly energy consumption data is a time series. Time series forecasting methods are divided into statistical (regression, extrapolation, exponential smoothing, etc.) and structural models (neural networks, fuzzy logic models, Markov chains, etc.). In this study, the mathematical model ARIMA (Integrated Moving Average Autoregression Model) is considered. This model is based on the Box-Jenkins methodology, which is an adaptive model. Electricity consumption of the metallurgical plant of ArcelorMittal Temirtau JSC was taken as the initial data. The initial time series is the weekly profile of the electrical load, and the daily load profile is taken as the forecasting horizon.

\section{Box-Jenkins methodology for building ARIMA model}

Electricity consumption of the metallurgical plant of ArcelorMittal Temirtau JSC was taken as the initial data. The initial time series is a weekly electric load profile, a daily load profile is taken as the forecasting horizon, the sampling period is one hour. ARIMA's approach to time series is that the stationarity of the series is assessed first. Various tests reveal the presence of unit roots and the order of integration of the time series (usually limited to the first or second order). Further, if necessary, the series is transformed by taking the difference of the corresponding order and some ARMA model is built for the transformed model, since it is assumed that the resulting process is stationary, in contrast to the original non-stationary process [1]. The time series forecasting algorithm based on the Box-Jenkins methodology consists of the following stages:

1) checking the time series for stationarity by building graphs of the time series, the correlation function and the private autocorrelation function;

2) estimation of the parameters of the ARIMA model;

3) forecasting and assessing the accuracy of the ARIMA model [2].

The choice of the parameters of the ARIMA model is reduced to a separate consideration of the AR component and the MA component. The AR component of the ARIMA model is a separate autoregressive model, in which the values of the time series at the moment are linearly dependent on the previous values of the same series. An autoregressive process of order $p$ for describing the time series of power consumption is defined as follows (1):

$$
x(t)=c+\sum_{i=1}^{p} a_{i} \cdot x_{t-i}+\varepsilon_{t},
$$

where $t$ - time series step,

$x(t)$ - power value of the electrical load at time $t$,

$c$ - constant coefficient that determines the power of the electrical load at the current time without the influence of external factors (by default it is equal to zero),

$i$ - time series delay from baseline,

$p$ - AR model order (defined as the maximum delay value from the original series),

$a_{1}, \ldots, a_{p}-\mathrm{AR}$ model coefficients describing the degree of dependence of the current value of the power of the electrical load on the past values,

$x_{t-i}-$ previous power values of the electrical load,

$\varepsilon_{t}-$ white noise.

The MA component of the ARIMA model is a separate moving average model, in which the current value of the time series is equal to the average value over a certain previous period of time. The moving average model of order $q$ has the form (2):

$$
x(t)=\sum_{j=0}^{q} b_{j} \cdot \varepsilon_{t-j},
$$

where $t$ - time series step,

$x(t)$ - power value of the electrical load at time $t$,

$i$ - time series delay from baseline,

$q$ - MA model order (defined as the maximum delay value from the original series),

$b_{1}, \ldots, b_{q}-$ MA coefficients of the model, describing the degree of dependence of the current value of white noise on the past values,

$\varepsilon_{t}-$ white noise. 
The Autoregressive Moving Average Model (ARMA) is one of the mathematical models used to analyze and forecast stationary time series in statistics. The ARMA model generalizes two simpler time series models - the autoregressive model (AR) and the moving average (MA) model. The $\operatorname{ARMA}(p, q)$ model, where $p$ and $q$ are integers specifying the order of the model, is the following process of generating a time series (3):

$$
x(t)=c+\sum_{i=1}^{p} a_{i} \cdot x_{t-i}+\sum_{i=1}^{q} b_{i} \cdot \varepsilon_{t-i}+\varepsilon_{t},
$$

where $t$ - time series step,

$x(t)$ - power value of the electrical load at time $t$,

$i$ - time series delay from baseline,

$c$ - constant coefficient that determines the power of the electrical load at the current time without the influence of external factors (by default it is equal to zero),

$p$ - AR model order (defined as the maximum delay value from the original series),

$q$ - MA model order (defined as the maximum delay value from the original series),

$a_{1}, \ldots, a_{p}-\mathrm{AR}$ model coefficients describing the degree of dependence of the current value of the power of the electrical load on the past values,

$b_{1}, \ldots, b_{q}-$ MA coefficients of the model, describing the degree of dependence of the current value of white noise on the past values,

$x_{t-i}$ - previous power values of the electrical load,

$\varepsilon_{t}-$ white noise.

The ARIMA model is an extension of ARMA models for non-stationary time series, which can be made stationary by taking differences of some order from the original time series. The $\operatorname{ARIMA}(p, d, q)$ model means that time series differences of order $d$ obey the $\operatorname{ARMA}(p, q)$ model.

The $\operatorname{ARIMA}(p, d, q)$ model for a non-stationary time series has the form (4):

$$
\Delta^{d} x(t)=c+\sum_{i=1}^{p} a_{i} \cdot \Delta^{d} x_{t-i}+\sum_{j=1}^{q} b_{j} \cdot \varepsilon_{t-j}+\varepsilon_{t},
$$

where $t$ - time series step,

$\Delta^{d}$ - operator of the time series difference of order $\mathrm{d}$, determined when converting the current time series of the power of the electrical load into a stationary form,

$x(t)$ - power value of the electrical load at time $t$,

$i$ - time series delay from baseline,

$c$ - constant coefficient that determines the power of the electrical load at the current time without the influence of external factors (by default it is equal to zero),

$p$ - AR model order (defined as the maximum delay value from the original series),

$q$ - MA model order (defined as the maximum delay value from the original series),

$a_{1}, \ldots, a_{p}-\mathrm{AR}$ model coefficients describing the degree of dependence of the current value of the power of the electrical load on the past values,

$b_{1}, \ldots, b_{q}-$ MA coefficients of the model, describing the degree of dependence of the current value of white noise on the past values,

$x_{t-i}-$ previous power values of the electrical load,

$\varepsilon_{t}-$ white noise [3].

The theoretical autocorrelation (ACF) and partial autocorrelation function (PACF) for the AR, MA and ARMA models are known and are different for each model. These differences between models are important to keep in mind when choosing models. Table 1 provides a description of the behaviour of ACF and PACF for AR, MA and ARMA models.

\begin{tabular}{|c|l|l|}
\hline Model type & \multicolumn{1}{|c|}{ ACF behaviour } & \multicolumn{1}{c|}{ PACF behaviour } \\
\hline $\operatorname{AR}(p)$ & Tails off gradually & Cuts off after $p$ lags \\
\hline $\operatorname{MA}(q)$ & Cuts off after q lags & Tails off gradually \\
\hline $\operatorname{ARMA}(p, q)$ & Tails off gradually & Tails off gradually \\
\hline
\end{tabular}

Table 1. Comparative data on the properties of ACF and PACF

To select the type of model (AR, MA, ARMA or ARIMA), you should build graphs of the initial time series, ACF and PACF and evaluate their behaviour [4]. 


\section{Checking the time series for stationarity}

The stationarity of the time series means that the probabilistic characteristics of the time series are unchanged over time. Unlike regular sample data, time series data is ordered. The study of the time series graph allows us to draw preliminary conclusions about stationarity. Figure 1 shows the hourly electricity consumption schedule of ArcelorMittal Temirtau JSC from January 1 to January 7, 2020. The absence of a trend gives a preliminary conclusion about stationarity, with the exception of the peak component on January 6.

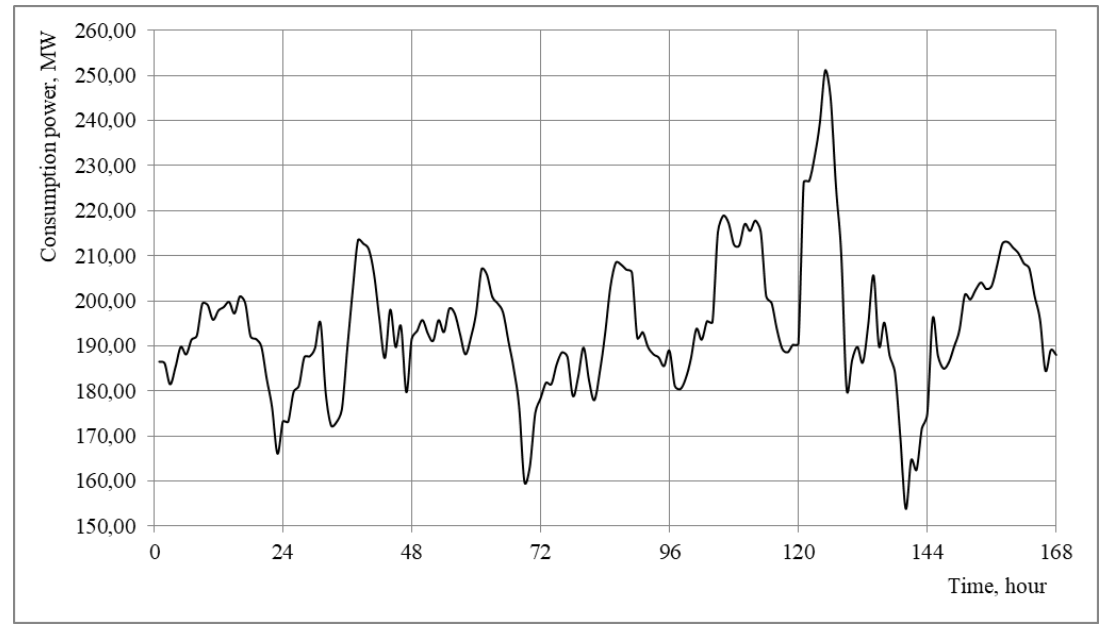

Fig. 1. Hourly power consumption chart for one week

The autocorrelation function (ACF) and the partial autocorrelation function (PACF) give more detailed characteristics of the time series, which make it possible to further evaluate the parameters of the ARIMA model. In particular, the ACF reports a correlation between points separated by different time delays, called lags [5].

In time series analysis, ACF shows the degree of linear statistical relationship between time series values. Numerically, the ACF is a sequence of correlation coefficients between the original series and its copy shifted by a given number of series intervals, called the lag $L(5)$ :

$$
f(L)=\sum_{L=0}^{n} r_{t, t-L}
$$

where $f(L)$ - autocorrelation function,

$n$ - number of time series levels,

$L$ - time series delay,

$r_{t, t-L}-$ correlation coefficient for lag $L$.

The graph of the dependence of the correlation coefficient on the lag is called a correlogram. It is an effective tool for studying the dynamic properties of time series [6].

The ACF is maximal at $L=0$, when the series is completely correlated with itself. Since the value of the autocorrelation coefficient varies from -1 to 1 , the ACF can only take values from the same range. The autocorrelation coefficient is determined as follows (6):

$$
r_{t, t-L}=\frac{\operatorname{cov}_{y_{t}, y_{t-L}}}{\sigma_{y_{t}} \cdot \sigma_{y_{t-L}}}=\frac{\sum\left(y_{t}-\bar{y}_{t}\right) \cdot\left(y_{t-L}-\bar{y}_{t-L}\right)}{\sqrt{\sum\left(y_{t}-\bar{y}_{t}\right)^{2} \cdot \sum\left(y_{t-L}-\bar{y}_{t-L}\right)^{2}}},
$$

where $r_{t, t-L}-$ correlation coefficient for lag $\mathrm{L}$,

$n$ - number of time series levels,

$L$ - time series delay,

$\operatorname{cov}_{y_{t}, y_{t-L}}$ - covariance coefficient,

$\sigma_{y_{t}}$ - standard deviation of the original time series,

$\sigma_{y_{t-L}}$ - lagged sample standard deviation,

$y_{t}-$ current value of the original time series,

$y_{t-L}-$ current sample value with lag,

$\bar{y}_{t}-$ mean of the original time series,

$\bar{y}_{t-L}$ - sample mean with lag $L$ [7]. 
If the series is affected by any long-term external factors, then this leads to the appearance of trends in the series and a cyclical component, which allows the ACF to be detected. Based on the type of the ACF correlogram, the following conclusions can be drawn:

- if the expressed maximum of the ACF correlogram turns out to be for the lag $L=k$, then the time series contains a cyclic component with a period of $k$;

- if the correlogram of the ACF does not have pronounced maxima, then the series does not contain a trend and a cyclical component, and a random component dominates in it [8].

Figure 2 shows the ACF of the investigated time series, which contains a break after 4 lags.

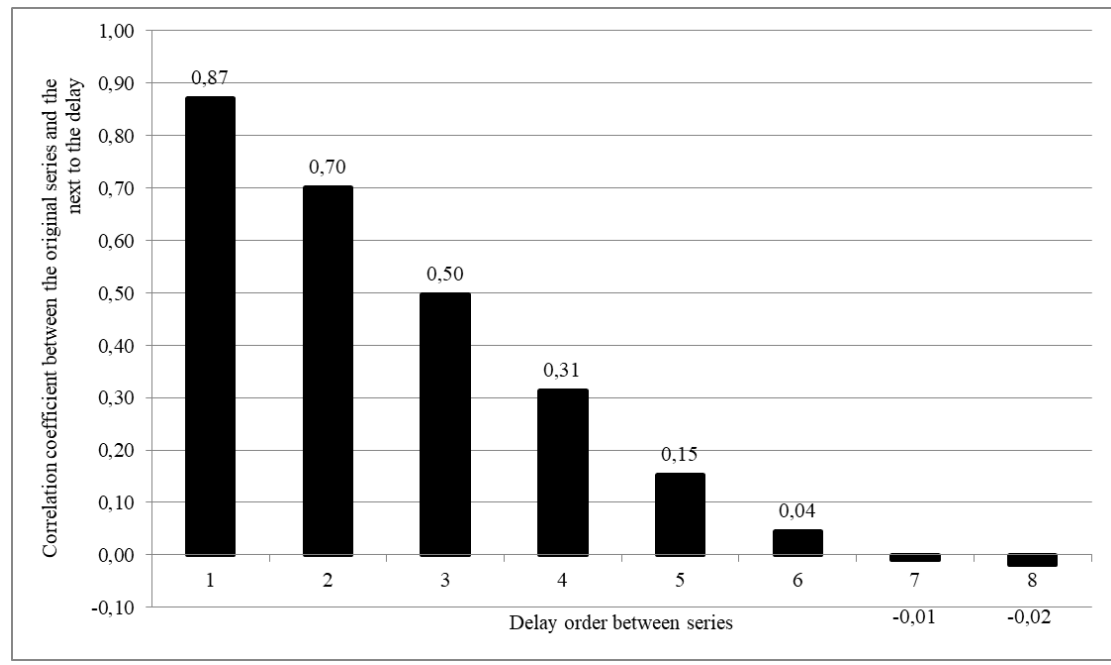

Fig. 2. Autocorrelation function of the time series of electricity consumption

Another way of preliminary estimation of the stationarity of the time series is the private autocorrelation function. Partial correlation coefficients between two random variables with a fixed third characterize the tightness of the relationship between these two values, cleared of the influence of the third variable. Therefore, if the paired correlation coefficient between the same two random variables turned out to be greater than the corresponding partial coefficient, then it is concluded that the third fixed value enhances the relationship between the studied values, i.e. the higher value of the pair coefficient is due to the presence of the third value. A lower value of the pair correlation coefficient in comparison with the corresponding partial indicates a weakening of the relationship between the studied values due to the action of the fixed value [9]. Figure 3 shows the PACF graph of the investigated time series, which contains a break after 3 lags.

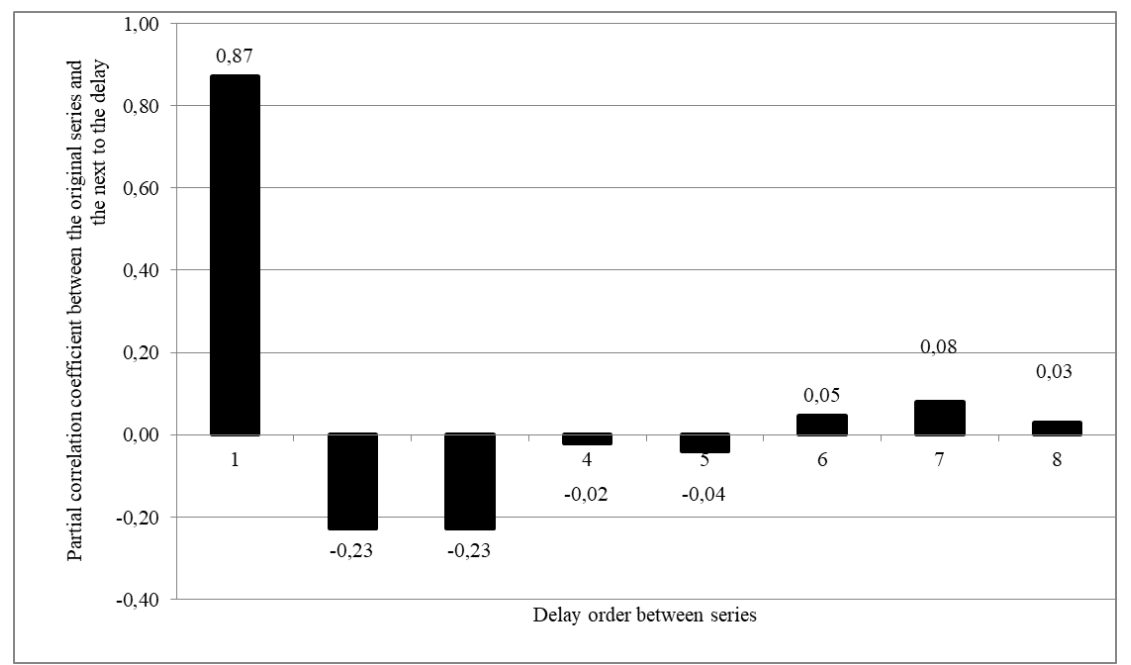

Fig. 3. Private autocorrelation function of the time series of electricity consumption

Based on the time series plots, ACF and PACF and theoretical data on the behaviour of the AR, MA and ARMA model, it can be concluded that the proposed model will have the form ARIMA $(p, 0, q)$ or ARMA $(p, q)$. Parameters $p$ and $q$ are selected experimentally. 


\section{Predicting and evaluating the accuracy of the ARIMA model}

Analysis of the time series graph indicates stationarity and the order of integration $d=0$, the ACF gives the assumed range of coefficients of the AR-component of the model $p=0 \ldots 4$, the analysis of the PACF gives the range of coefficients $q=0 \ldots 3$. It is necessary to build a forecast for January 8,2021 . When assessing the predictive ability of the model, the parameter of the root-mean-square estimate RMSE was used (7):

$$
R M S E=\sqrt{\sum_{i=1}^{n} \frac{\left(y_{i}-\hat{y}_{i}\right)^{2}}{n}},
$$

where $y_{i}-$ actual value of the time series,

$\hat{y}_{i}$ - time series predicted value,

$n$ - time series length [10].

Table 2 shows an estimate of the possible combinations of $p, d, q$ and the RMSE [11] coefficients of the constructed models.

\begin{tabular}{|c|c|}
\hline Model type & RMSE \\
\hline ARIMA(3,0,1) & 0.0641 \\
\hline ARIMA(3,0,2) & 0.0661 \\
\hline ARIMA(3,0,3) & 0.0646 \\
\hline ARIMA $(4,0,1)$ & 0.0679 \\
\hline ARIMA $(4,0,2)$ & 0.0644 \\
\hline ARIMA(4,0,3) & 0.0622 \\
\hline
\end{tabular}

Table 2. Assessing the accuracy of alternative ARIMA models

The ARIMA model $(4,0,3)$ has the best predictive power, as suggested by ACF and PACF charts.

\section{Conclusion}

The management of the electricity consumption schedule at large enterprises in Kazakhstan with various types of production is an urgent task. To predict the consumption of electric energy of ArcelorMittal Temirtau JSC on the basis of retrospective data, an adaptive model of the ARIMA type was developed, built according to the Box-Jenkins methodology. The model is highly flexible, and the disadvantages associated with its rather low versatility are compensated by the presence of detailed time series of power consumption of an energy-intensive metallurgical plant. Its advantages include the fact that for its construction it is sufficient to use the data of only the original time series.

It was found that for the forecast horizon of power consumption per day with a sampling step of one hour, it is optimal to consider a sample with hourly data for the week proceeding the day under study. The dynamics of changes in the power consumption of the electric load graph, the gradual attenuation of the ACF and PACF allows us to conclude about the stationary nature of the power consumption process. When analysing the weekly profile of electrical load, a functional dependence of time data with a delay of up to 4 orders of magnitude was revealed with a confidence interval of $95 \%$ (high correlation coefficient with a delay: at 1 hour -0.8 and 2 hours -0.7 ; significant correlation coefficient with a delay in: 3 hours -0.5 and 4 hours -0.31 ).

The ARIMA model in the presence of an initial time series can be applied to predict the power consumption of large energy-intensive industries of the mining and metallurgical complex, taking into account the fact that there is a detailed methodology, following which you can choose a model that is most suitable for each specific enterprise. The models have a very clear mathematical and statistical basis, which makes them one of the most promising of the whole variety of options for predicting the power consumption of large enterprises with various types of production. For this model, the weekly profile of the electrical load in winter was taken as retrospective data. In the future, it is planned to consider the monthly profile of the electrical load. It will also be possible to review data for other seasons, identify the seasonal component and obtain a SARIMA model.

\section{References}

[1] Kazantsev V. P.; Petrochenkov A. B.; Romodin A. V. \& Khoroshev N. I. (2011). Some aspects of technology of use of electrical objects on the basis of methods of short-term forecasting of technical condition. Russian Electrical Engineering, Vol. 82, No. 11, pp. 600-606.

[2] Kuntush, Y. V.; Breido, J. V. \& Zyuzev, A. M. (2020). Developing a mathematical model of a horizontal looper taking into account the features of a steel strip. EAI Endorsed Transactions on Energy Web, Vol. 7, No. 28, E11. 
[3] Taylor J. W. \& McSharry P. E. (2008). Short-Term Load Forecasting Methods: An Evaluation Based on European Data. IEEE Transactions on Power Systems, Vol. 22, pp. 2213-2219.

[4] Mindaugas C. (2020). Lithuanian electricity market price forecasting model based on univariate time series analysis. Energetika, Vol. 66, No. 1, pp. 39-46.

[5] Kaytez F.; Taplamacioglu M. C.; Cam E. \& Hardalac F. (2015). Forecasting electricity consumption: A comparison of regression analysis, neural networks and least squares support vector machines. International Journal of Electrical Power \& Energy Systems, Vol. 67, pp. 431-438.

[6] Lee N. C.; Leal V. M. S. \& Dias L. C. (2018). Identification of objectives for national energy planning in developing countries. Energy Strategy Reviews, Vol. 21, pp. 218-232.

[7] Lopez M.; Valero S.; Rodriguez A.; Veiras I. \& Senabre C. (2018). New online load forecasting system for the Spanish Transport System Operator. Electric Power Systems Research, Vol. 154, pp. 401-412.

[8] Jimenez J.; Donado K. \& Quintero C. G. (2017). A methodology for short-term load forecasting. IEEE Latin America Transactions, Vol. 15, No. 3, pp. 400-407.

[9] Chinnathambi R. A.; Mukherjee A.; Campion M.; Salehfar H.; Hansen T. M.; Lin J. \& Ranganathan P. (2019). A multi-stage price forecasting model for day-ahead electricity markets. Forecasting, Vol. 1, pp. 26-46.

[10] Breido J. V.; Kalinin A. A. \& Lissitsyn D. V. (2018). Algorithms of energy efficient control of electric technological complex for autonomous heat supply. EAI Endorsed Transactions on Energy Web, Vol. 18, No. 19, e2.

[11] Kim B. S. \& Kim T. G. (2019). Cooperation of simulation and data model for performance analysis of complex systems. International Journal of Simulation Modelling, Vol. 18, No. 4, pp. 608-619. 